Discussiones Mathematicae

\title{
GENERALIZED HAMMING GRAPHS: SOME NEW RESULTS
}

\author{
AMARI BEDRANE \\ AND \\ BERRACHEDI ABDELHAFID \\ LIFORCE Laboratery \\ RO Department, USTHB, Algiers, Algeria \\ e-mail: bedrane.amari@gmail.com
}

\begin{abstract}
A projection of a vertex $x$ of a graph $G$ over a subset $S$ of vertices is a vertex of $S$ at minimal distance from $x$. The study of projections over quasi-intervals gives rise to a new characterization of quasi-median graphs.

Keywords: generalized median graphs, Hamming graphs, quasi-median graphs, quasi-Hilbertian graphs.
\end{abstract}

2010 Mathematics Subject Classification: 2010 MSC 05C75, 05C12.

\section{REFERENCES}

[1] N. Badji and A. Berrachedi, Generalized median graphs, in: Actes du Colloque sur l'opt. et les sys. d'infor., COSI'04 (UMM Tizi Ouzou, Algerie, 2004) 216-225.

[2] H.J. Bandelt, Retracts of hypercubes, J. Graph Theory 8 (1984) 501-510. doi:10.1002/jgt.3190080407

[3] H.J. Bandelt, H.M. Mulder and E. Wilkeit, Quasi-median graphs and algebras, J. Graph Theory 18 (1994) 681-703. doi:10.1002/jgt.3190180705

[4] A. Berrachedi, A new characterization of median graphs, Discrete Math. 128 (1994) 385-387. doi:10.1016/0012-365X(94)90128-7

[5] A. Berrachedi and M. Mollard, Median graphs and hypercubes, some new characterizations, Discrete Math. 208/209 (1999) 71-75. doi:10.1016/S0012-365X(99)00063-1 
[6] F.R.K. Chung, R.L. Graham and M.E. Saks, A dynamic location problem for graphs, Combinatorica 9 (1989) 111-131.

doi:10.1007/BF02124674

[7] W. Imrich and S. Klavžar, Product Graphs: Structure and Recognition (John Wiley \& Sons, New York, 2000).

[8] H.M. Mulder, n-cubes and median graphs, J. Graph Theory 4 (1980) 107-110. doi:10.1002/jgt.3190040112

[9] H.M. Mulder, The Interval Function of a Graph (Mathematical Centre Tracks 132, Mathematish Centrum, Amesterdam, 1980).

[10] L. Nebeský, Algebraic properties of Husimi trees, Časopis Pešt. Math. 107 (1982) 116-123.

[11] E. Wilkeit, The retracts of Hamming graphs, Discrete Math. 102 (1992) 197-218. doi:10.1016/0012-365X(92)90054-J

Received 13 June 2016

Revised 16 January 2017

Accepted 16 January 2017 